\title{
COMPORTAMIENTO PRODUCTIVO DE VACAS LECHERAS ALIMENTADAS CON Moringa oleifera FRESCO O ENSILADO: EFECTO SOBRE PRODUCCIÓN, COMPOSICIÓN Y CARACTERÍSTICAS ORGANOLÉPTICAS DE LECHE Y QUESO
}

\section{PRODUCTIVE BEHAVIOR OF DIARY COWS FEEDED Moringa oleifera FRESH OR ENSILED: EFFECT ON YIELD, COMPOSITION AND ORGANOLEPTIC CHARACTERISTICS OF MILK AND CHEESE}

\author{
Rodríguez-Pérez Rosario de la Concepción ${ }^{1}$, Reyes-Sánchez Nadir ${ }^{2}$, Mendieta-Araica Bryan ${ }^{3}$ \\ ${ }^{1}$ MSc en Agroecología, Universidad Nacional Agraria \\ ${ }^{2} \mathrm{PhD}$ en Ciencia Animal, Universidad Nacional Agraria \\ ${ }^{3} \mathrm{PhD}$ en Ciencia Animal, Universidad Nacional Agraria, bryan.mendieta@una.edu.ni
}

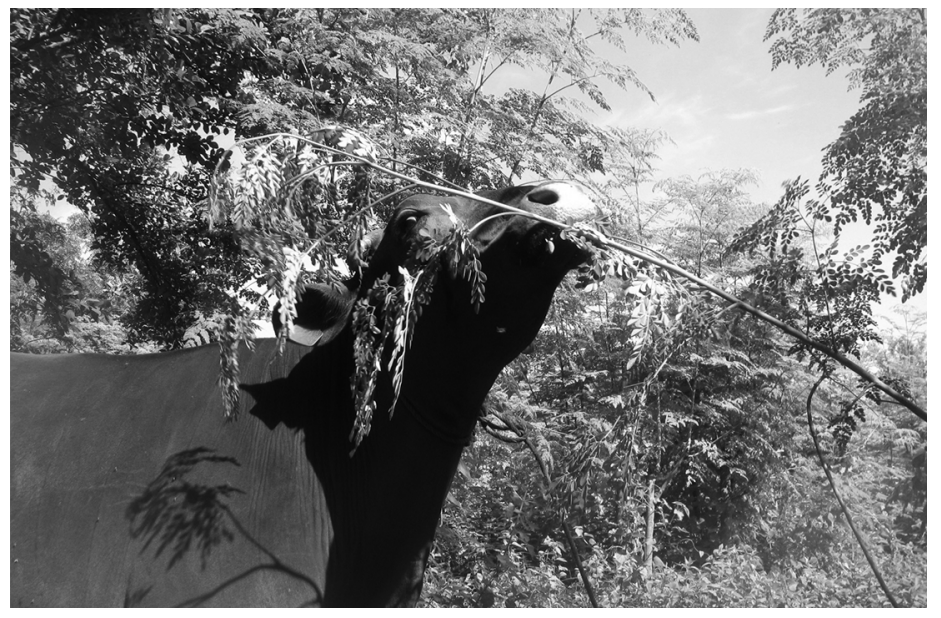

\section{RESUMEN}

Moringa oleifera fresca o ensilada, se comparó con una dieta control basada en Pennisetum purpureum cv CT-115 + concentrado comercial como dieta única para vacas lecheras. Se evaluó el efecto de las dietas experimentales sobre el consumo y la digestibilidad, la producción y composición de la leche, y las características organolépticas de leche y queso. Seis vacas lecheras fueron utilizadas en un experimento cuadrado latino $3 \times 3$, con dos repeticiones. Las dietas de Moringa fueron isocalóricas respecto a la dieta control. El consumo de materia seca (MS) de los tratamientos de Moringa fue significativamente mayor $(P<0.05)$ que el control. Moringa fresca tuvo el mayor consumo $(P<0.001)$ de materia orgánica $(\mathrm{MO})$, proteína bruta $(\mathrm{PB})$, fibra detergente neutra (FDN), fibra detergente ácido (FDA) en comparación con la dieta de control. Los mayores coeficientes de digestibilidad $(P<0.05)$ se manifestaron en el tratamiento control respecto a los tratamientos de Moringa (fresca o ensilada), con la excepción de la digestibilidad de la PB. La producción de leche del tratamiento de Moringa ensilado fue ligeramente inferior (9\%) respecto a los otros dos tratamientos. La composición de la leche fue similar entre todos los tratamientos. Sin embargo, la leche del tratamiento de Moringa fresca presentó un sabor y aroma a hierba, significativamente diferente $(P<0.001)$ de los otros dos tratamientos, a pesar de que era normal en color y apariencia. No se observaron diferencias organolépticas entre la leche del tratamiento control y el tratamiento de ensilaje de Moringa. Igual comportamiento se encontró en el queso elaborado a partir de la leche de los tres tratamientos. El análisis financiero favorece a los tratamientos de Moringa. La conclusión es que el ensilaje de Moringa se puede utilizar para alimentar vacas lecheras en grandes cantidades para producir cantidades similares y misma producción de leche, características organolépticas. calidad de la leche que las dietas convencionales.

Palabras clave: Moringa oleifera, ensilaje, producción de leche.

\section{ABSTRACT}

Moringa oleiferafresh or ensiled was compared with a control diet based on Pennisetum purpureumcv CT-115 + commercial concentrate as basal diet for dairy cattle. The effect of experimental diets on intake and digestibility, milk yield and composition, and organoleptic characteristics of milk and cheese was evaluated. Six lactating dairy cows were used in a changeover $3 \times 3$ latin square trial, replicated twice. Moringa diets were isocaloric with respect to the control diet. The dry matter (DM) intake of Moringa treatment was significantly higher $(\mathrm{P}<0.05)$ than the control. Intake of organic matter $(\mathrm{OM})$, crude protein $(\mathrm{CP})$, neutral detergent fiber (NDF), acid detergent fiber (ADF) of Fresh Moringa were higher $(\mathrm{P}<0.001)$ compared with the control diet. Higher digestibility coefficient of digestibility $(\mathrm{P}<0.05)$ were obtained in control treatment compared with Moringa treatments (fresh or ensiled), with the exception of the digestibility of CP. Milk yield of Moringa silage treatment was slightly lower (9\%) than the other two treatments. The milk composition was similar among all treatments. However, milk fresh Moringa treatment had a grassy flavor and aroma, significantly different $(\mathrm{P}<0.001)$ from the other two treatments, although it was normal in color and appearance. There were no differences in organoleptic characteristics between milk from the control treatment and treatment of silage Moringa. Similar pattern was found in cheese made from milk of three treatments. Financial analysis favors the treatment of Moringa. The conclusion is that Moringa silage can be used to feed dairy cows in large amounts to produce the similar quantity and same quality of milk than conventional diets.

Keywords: Moringa oleifera, ensiled, milk yield. 
$\mathrm{L}$ a producción ganadera es una parte muy importante del sector agropecuario de muchos países en vías de desarrollo, representando hasta el $40 \%$ del producto nacional bruto (Steinfeld et al., 2006). El sector lechero, en particular, es de una importancia creciente. Por ejemplo, la producción de leche en Latino América incrementó en 12\% entre el 2003 y el 2007 (FAO, 2010).

Aunque ocurren variaciones regionales entre Centro y Sur América, es justo describir que los sistemas lecheros intensivos en Latino América consisten en pequeños a medianas fincas con 10-17 ha y entre 15-21 vacas (Holmann et al., 2003). Esas fincas generalmente practican alimentación en el establo, proveyendo principalmente Pennisetum purpureum como forraje y melaza o subproductos, materias primas o concentrados disponibles localmente. La razas mas populares son la Pardo Suizo y la Holstein Fresian y sus cruces con ganado índico, las producciones promedios para las fincas aquí mencionadas es de aproximadamente $16 \mathrm{~kg}$ de leche $\mathrm{vaca}^{-1}$ día $^{-1}$ (rango 5 -27 kg). Aun cuando los forrajes disponibles son usualmente de baja calidad nutricional, son muy pocos los productores que pueden comprar concentrados para suplementar durante las épocas de escasez debido a su alto costo.

Aun cuando en regiones tropicales como la nuestra, hay un enorme potencial para la producción de biomasa, los forrajes son abundantes solo durante la época lluviosa, el ensilaje se ha usado para sobrellevar la escasez que impera durante la época seca, sin embargo, dado que los pastos tropicales maduran con rapidez, el ensilado obtenido no siempre cuenta con buena calidad nutricional, por lo que el problema de encontrar una solución barata a la escasez de alimento aun persiste.

El potencial de los sistemas agroforestales para suplir animales con alimento de alta calidad ha sido ampliamente reconocido y sus cualidades de tolerancia a la sequía, altos contenidos de proteína en su follaje y adaptación a muchos sistemas de manejo los hacen interesantes para incorporarlos a los sistemas de producción lechero; entre las distintas especies de árboles y arbustos conocidas y usadas en estos sistemas, destaca marango (Moringa oleifera) por su alto contenido de proteína bruta en las hojas, altos coeficientes de digestibilidad e ínfimos contenidos de factores antinutricionales. Su efecto positivo cuando se incluye en dietas para animales ha sido reportado (Manhet al., 2005). Por otro lado, su ensilabilidad ha sido ya reportada (Mendieta-Araica et al., 2009), sin embargo, dietas basadas enteramente en follaje o ensilaje de marango no han sido estudiadas en vacas lecheras, por lo que el objetivo de este estudio es evaluar el rendimiento y composición de la leche, la digestibilidad de la dieta y especial atención se presta a las características organolépticas de la leche y el queso elaborado a partir de la anterior.

\section{MATERIALES Y METODOS}

Localización y duración del experimento. El estudio se realizó en la finca Santa Rosa de la Universidad Nacional Agraria (UNA) en Managua, Nicaragua, localizada a los $12^{\circ}$ $08^{\prime} 33$ " de latitud norte y $86^{\circ} 10^{\prime} 31^{\prime}$ " longitud oeste; con una temperatura media anual de $26.9^{\circ} \mathrm{C}$, precipitación media anual de $1119.8 \mathrm{~mm}$ y humedad relativa del $72 \%$ y con una marcada época seca (noviembre a mayo) (INETER, 2006). El ensayo estuvo comprendido entre el 19 de julio y el 11 octubre del 2009.

Preparación de los alimentos. Antes del inicio del experimento, las áreas de Pennisetum purpureum $c v$. CT115 y marango fueron divididas en parcelas, realizando corte de uniformidad en intervalos regulares de 45 días. El P.purpureum cv. CT-115, fue cosechado y posteriormente picado en partículas de aproximadamente dos $\mathrm{cm}$ de longitud en una picadora estacionaria y ofrecido de forma fresca a las vacas. Para el tratamiento de Marango fresco se recolectaron diariamente ramas frescas, las mismas separadas en fracciones, utilizando solamente la fracción fina (hojas y pecíolos $\leq 5 \mathrm{~mm}$ de diámetro). Para el ensilaje de Marango, la fracción fina fue picada y se le agregó melaza (5\% del peso fresco) para facilitar el proceso de fermentación del material a ensilar y luego colocada en bolsas de polietileno (55*97 $\mathrm{cm})$. Las bolsas fueron llenadas en capas sucesivas de 30 $\mathrm{cm}$, compactadas por presión manual, cerradas y finalmente selladas con ayuda de una banda elástica para evitar la entrada de aire. Se elaboraron 180 bolsas (silos) con pesos aproximados de $45 \mathrm{~kg}$. Posteriormente fueron almacenadas sobre polines de madera en una bodega con piso de concreto durante un periodo de 120 días. El concentrado fue elaborado en la planta procesadora de alimento de la UNA con ingredientes disponibles comercialmente. Este concentrado estaba conformado de los siguientes ingredientes: pulido de arroz, sorgo, harina de soya, melaza, carbonato de calcio, harina de maní, cloruro de sodio en las siguientes proporciones: 41-30-20-6-1.5-1-0.5. La melaza de caña de azúcar fue adquirida por medio de un distribuidor local de productos agrícolas.

Manejo de los animales. Se utilizaron seis vacas lecheras Pardo Suizo*Brahman, con un peso vivo promedio de $416( \pm 50) \mathrm{kg}$, que se encontraban entre la segunda y tercer lactancia y en la cuarta semana de lactación al iniciar el experimento. Los animales fueron pesados al inicio del ensayo y alojados individualmente en un establo bien ventilado. Antes de iniciar el ensayo, a los animales se les aplicó vitaminas A (625,000 UI), D 3 (125,000 UI) y E (125 UI), Ivermectina $1 \%$ para control de parásitos externos e internos y vacuna contra ántrax. A todos los animales se les garantizó acceso al agua ad libitum y sales minerales acorde a sus requerimientos. Las vacas se ejercitaron diariamente en un área común, aprovechando el momento de la limpieza de 
los cubículos individuales, excepto durante los periodos de recolección de heces.

Tratamientos. Las seis vacas lecheras utilizadas en el experimento fueron distribuidas en tres tratamientos. El diseño experimental utilizado fue un Doble Cuadrado Latino 3*3 según descrito por Patterson and Lucas (1962). Cada período experimental tuvo una duración de cuatro semanas de las cuales las dos primeras semanas eran de adaptación a los tratamientos y las otras dos semanas de evaluación y recolección de datos de producción de leche y consumo de alimentos. La digestibilidad aparente de cada nutriente fue estimada en la última semana de cada período experimental mediante el método de colecta total de heces

Los tratamientos evaluados fueron:

- T1: Forraje fresco P. purpureum cv. CT-115 + concentrado comercial

- T2: Forraje fresco de marango $+1 \mathrm{~kg}$ de melaza.

- T3: Ensilaje de marango $+1 \mathrm{~kg}$ de melaza.

Los datos fueron analizados utilizando el Proc GLM del Software SAS versión 9.1.2 (SAS $\left.{ }^{\circledR}, 2004\right)$. El procedimiento de comparación de medias por la prueba de Tuckey fue utilizado cuando las diferencias entre tratamientos eran significativas al $\mathrm{P}<0.05$.

El modelo matemático utilizado fue el siguiente:

$\mathrm{Y}_{\mathrm{ijk}}=\mu+\mathrm{P}_{\mathrm{i}}+\mathrm{C}_{\mathrm{j}}+\mathrm{T}_{\mathrm{k}}+\varepsilon_{\mathrm{ijk}}$

Donde $\mu=$ media general; $\mathrm{P}_{\mathrm{i}}=$ efecto fijo de período ( $\mathrm{i}=1,2$, 3); $\mathrm{C}_{\mathrm{j}}=$ efecto aleatorio de vaca dentro del cuadrado $(\mathrm{j}=1$, $2 \ldots, 6) ; \mathrm{T}_{\mathrm{k}}=$ efecto fijo de tratamiento $(\mathrm{k}=1,2,3)$; $\epsilon_{\mathrm{ijk}}=$ error residual aleatorio.

El efecto residual de los tratamientos y las interacciones entre los efectos fueron analizados y removidos del modelo debido a la falta de significancia $(\mathrm{P}>0.1)$.

Los datos de las encuesta para leche y queso fueron analizados por medio de pruebas no paramétricas utilizando la prueba de Kruskal Wallis.

La cantidad total de alimento ofrecido $(\mathrm{kg}$ materia seca (MS) vaca ${ }^{-1} \mathrm{dí}^{-1}$ ) fue calculada utilizando la fórmula del NRC (1988).

El T1 (control) fue calculado para cumplir con los requerimientos de proteína bruta $(\mathrm{PB})$ y energía metabolizable (EM) (NRC, 1988); proporcionó 60\% del consumo de materia seca calculado en base a forraje fresco y el $40 \%$ restante mediante concentrado comercial. Los tratamientos con marango (T2 y T3) se calcularon que fueran isocalóricos con respecto al tratamiento control (T1), se usó un kilogramo de melaza uniformemente distribuida; a su vez la melaza contribuyó a ajustar el nivel energético de los tratamientos.
Los forrajes fueron ofertados a los animales individualmente en comederos separados, dos veces al día, a las 07:00 h y a las 17:00 h. El concentrado del tratamiento control fue suministrado de forma individual durante el ordeño, a las 05:00 h y 16:00 h, En el caso de los tratamientos dos y tres la melaza se fraccionó y se mezcló uniformemente con el forraje, cada vez que estos fueron ofrecidos. El contenido de materia seca de los forrajes ofrecidos fue determinado diariamente utilizando un horno de microondas de acuerdo al proceso descrito por Undersander et al., (1993). Los alimentos ofrecidos y los rechazos ocasionales fueron pesados diariamente, tomando muestras de un kilogramo del alimento ofrecido por vaca día ${ }^{-1}$ el que se congeló a $-18{ }^{\circ} \mathrm{C}$. $\mathrm{Al}$ final de cada período experimental las muestras fueron descongeladas y agrupadas para obtener una muestra por cada periodo y sometidas a análisis químico. Igualmente al concentrado se le tomó una muestra de un kilogramo de forma semanal y se envió al laboratorio para su análisis químico.

Las vacas se ordeñaron manualmente dos veces por día, a las 05:00 h y a las 16:00 h, los registros de producción de leche se obtuvieron del pesaje diario de esta. Los últimos tres días de cada período se tomaron muestras de leche por vaca, las que fueron refrigeradas y enviadas al laboratorio para su respectivo análisis químico.

Digestibilidad. En la última semana de cada período del experimento, las heces de cada vaca fueron colectadas diariamente de forma manual y colocadas en un contenedor con tapa, la recolección de cada 24 h se pesó y mezcló de forma homogénea tomándose una muestra correspondiente al $5 \%$ del total, estas se colocaron en bolsas individuales, las que fueron identificadas y congeladas. Cuando la colecta de heces se completó, las sub-muestras de cada vaca se descongelaron y se mezclaron para obtener una muestra homogénea por vaca y por período. Aproximadamente $300 \mathrm{~g}$ de la muestra compuesta de cada animal fue llevada al laboratorio para análisis químico. Los resultados de los análisis de las heces y del alimento más los datos del consumo fueron utilizados para estimar la digestibilidad aparente.

Elaboración de queso. Para la elaboración de quesos provenientes de los tratamientos se tomó aproximadamente una cantidad de 10 litros de leche por tratamiento. De forma individual cada muestra de leche fue depositada en un recipiente para su debido reposo, posteriormente a éste se le agrego fermento $\left(1 \mathrm{ml} 101\right.$ leche $\left.^{-1}\right)$, se agitó durante dos minutos para su homogenización y luego se dejó reposar 45 minutos; una vez conformada la cuajada esta fue cortada con ayuda de liras de forma horizontal y vertical para el desuerado, finalmente se le agregó 3\% del peso fresco de sal y se colocó en moldes de 0.5 a $1 \mathrm{~kg}$, para su prensado durante $24 \mathrm{~h}$. 
Análisis químicos y pruebas organolépticas. Las muestras secas de los alimentos ofrecidos y rechazos ocasionales fueron molidas y pasadas por un tamiz de un mm y almacenadas en recipientes de vidrios para posteriores análisis químicos. La MS y cenizas fueron analizadas según el procedimiento de la AOAC (1990). La concentración de nitrógeno total fue determinado utilizando el método de Kjeldahl (AOAC, 1984) y la concentración de proteína bruta fue calculada mediante la fórmula $\mathrm{PB}=\%$ de nitrógeno total $* 6.25$ (factor de ajuste de la proteína vegetal). Los contenidos de fibra neutro detergente (FND) y fibra ácido detergente (FAD) fueron analizados según lo descrito por Van Soest et al., (1991) utilizando sulfito de sodio.

La energía metabolizable (EM) de los forrajes fue calculada usando la ecuación de Lindgren (1979). Para determinar la energía metabolizable contenida en el concentrado se utilizó el análisis de Weende descrito por MDonald et al., (1988).

A las heces recolectadas por cada periodo se le realizaron análisis de $\mathrm{MS}, \mathrm{PB}$, ceniza; FND, FDA.

El contenido de nitrógeno en leche fue determinado por el método de Kjeldahl y el contenido de proteína en la leche fue calculado mediante la fórmula $\mathrm{PB}=\%$ de nitrógeno total * 6.38 (factor de ajuste a la proteína de la leche), la concentración de grasa en la leche por el método de Babcock (AOAC, 1984), y los sólidos totales y caseína fueron analizados de acuerdo al procedimiento descrito por la AOAC (1984).

Prueba organoléptica. Durante el último periodo del ensayo se realizó la evaluación organoléptica de la leche y queso mediante la metodología propuesta por Pastor et al., (2008). Ésta metodología permitió medir reacciones de respuestas desde la aceptación hasta el rechazo de los alimentos.

Análisis económico. Con la finalidad de comparar los costos de cada dieta así como los beneficios económicos que existen al sustituir una por otra, se realizó un análisis de presupuestos parciales con la metodología sugerida por Pérez (1993).

\section{RESULTADOS Y DISCUSIÓN}

Composición química de los ingredientes. Marango (fresco y ensilado) tuvo altas concentraciones de PB (241.2-217.7 g $\mathrm{kg}^{-1} \mathrm{MS}$ ) y una baja concentración de FDN en comparación con el $P$. purpureum $c v$. CT-115. El contenido de PB del concentrado es lo usual en alimentos comerciales balanceados para vacas lecheras en Centro América (tabla 1).

Tabla 1. Composición química de los ingredientes

\begin{tabular}{lrrrrr}
\hline Nutrientes & $\begin{array}{c}\text { P. purpureum } \\
\text { CT-1151 }\end{array}$ & $\begin{array}{c}\text { Follaje de } \\
\text { de marango }\end{array}$ & $\begin{array}{c}\text { Ensilaje de } \\
\text { marango }^{1}\end{array}$ & Melaza $^{1}$ & $\begin{array}{c}\text { Concentrado } \\
\text { comercial }^{1}\end{array}$ \\
\hline${\text { MS }\left(\mathrm{g} \mathrm{kg}^{-1}\right) \mathrm{g} \mathrm{kg}^{-1} \mathrm{MS}}^{153.40(17.30)}$ & $193.40(01.20)$ & $267.40(09.80)$ & $727.00(0.10)$ & $844.94(10.40)$ \\
PB & $107.70(02.00)$ & $241.20(05.20)$ & $217.70(05.70)$ & $21.70(0.30)$ & $184.00(01.58)$ \\
FDN & $506.50(17.30)$ & $365.20(06.10)$ & $354.80(17.90)$ & nd & $90.6003 .13)$ \\
FDA & $289.40(05.50)$ & $319.70(24.40)$ & $326.00(22.10)$ & nd & $58.20(11.20)$ \\
Lignina & $255.80(07.20)$ & $313.50(16.90)$ & $310.30(07.20)$ & nd & $43.20(04.49)$ \\
Cenizas & $180.80(00.30)$ & $92.80(03.20)$ & $116.10(03.20)$ & $27.80(0.10)$ & $76.82(03.98)$ \\
EM Mcal & $2.03(00.02)$ & $2.60(00.36)$ & $2.58(00.41)$ & $2.19(0.00)$ & $3.49(00.03)$ \\
No muestras & 6 & 6 & 6 & 6 & 6
\end{tabular}

MS: Materia seca; PB: Proteína Bruta; FDN: Fibra detergente neutra; FDA: Fibra detergente ácida; EM: Energía metabolizable; nd: no determinada; ${ }^{1}: \mu$ y SD de cada uno de los componentes de las dietas.

Los productores comúnmente suministran $P$. purpureum como dieta basal a las vacas lecheras, sin embargo, este madura rápidamente con la consiguiente reducción en la concentración de proteína bruta y la necesidad de suplementar la dieta basal con concentrados comerciales. Estudios previos han incluido marango en la dieta de vacas lecheras como forraje para reducir la necesidad de otros suplementos proteicos (Reyes-Sánchez et al., 2006). En el presente estudio, marango constituyo todo el forraje de la dieta sin suplemento proteínico y solo complementado con un $\mathrm{kg}$ de melaza.
Consumo de alimento y digestibilidad aparente. Consumo y digestibilidad aparente de los tratamientos, muestran diferencias $(\mathrm{P}<0.05)$ para materia seca, proteína bruta, materia orgánica y fibra ácido detergente entre el control y los tratamientos de marango. Respecto a fibra neutro detergente el tratamiento control tuvo un consumo ligeramente superior a los tratamientos de marango. Lignina presentó diferencias significativas $(\mathrm{P}<0.01)$ entre los tratamientos. EM presentó diferencias $(\mathrm{P}<0.05)$ entre marango ensilado y los otros dos tratamientos. 
Tabla 2. Consumo y digestibilidad aparente de P. purpureum CT-115 + concentrado comercial, marango fresco y ensilaje de marango

\begin{tabular}{|c|c|c|c|c|}
\hline \multirow[t]{2}{*}{ Nutrientes } & \multicolumn{3}{|c|}{ Tratamientos } & \multirow[b]{2}{*}{$\begin{array}{c}\text { Error } \\
\text { estándar }\end{array}$} \\
\hline & $\begin{array}{l}\text { P. purpureum } \\
\text { CT-115 + } \\
\text { concentrado } \\
\text { comercial }\end{array}$ & $\begin{array}{l}\text { Marango } \\
\text { fresco }\end{array}$ & $\begin{array}{c}\text { Ensilaje } \\
\text { de marango }\end{array}$ & \\
\hline$\overline{\mathrm{MS}}$ & $11.09 \mathrm{~b}$ & $11.20 \mathrm{ab}$ & $11.29 \mathrm{a}$ & 0.04 \\
\hline $\mathrm{MO}$ & $9.7 \mathrm{c}$ & $10.22 \mathrm{a}$ & $10.06 \mathrm{~b}$ & 0.03 \\
\hline PB & $1.54 \mathrm{~b}$ & $2.48 \mathrm{a}$ & $2.42 \mathrm{a}$ & 0.06 \\
\hline FDN & $3.77 \mathrm{a}$ & $3.73 \mathrm{ab}$ & $3.65 \mathrm{~b}$ & 0.01 \\
\hline FDA & $2.18 \mathrm{~b}$ & $3.26 \mathrm{a}$ & $3.40 \mathrm{a}$ & 0.02 \\
\hline Lignina & $1.89 \mathrm{c}$ & $3.38 \mathrm{a}$ & $3.19 \mathrm{~b}$ & 0.01 \\
\hline EM Mj día ${ }^{-1}$ & $28.99 \mathrm{a}$ & 28.71 a & $28.52 \mathrm{~b}$ & 0.1 \\
\hline MS & 0.76 a & $0.69 \mathrm{~b}$ & $0.54 \mathrm{c}$ & 1.22 \\
\hline PB & $0.77 \mathrm{a}$ & $0.78 \mathrm{a}$ & $0.74 \quad \mathrm{a}$ & 0.88 \\
\hline MO & $0.77 \mathrm{a}$ & $0.70 \mathrm{~b}$ & $0.68 \mathrm{~b}$ & 0.64 \\
\hline FDN & $0.59 \mathrm{a}$ & $0.54 \mathrm{~b}$ & $0.49 \mathrm{~b}$ & 1.14 \\
\hline FDA & $0.64 \mathrm{a}$ & $0.60 \mathrm{a}$ & $0.56 \mathrm{~b}$ & 0.94 \\
\hline
\end{tabular}

MS: Materia seca; MO: Materia orgánica; PB: Proteína Bruta; FDN: Fibra detergente neutra FDA: Fibra detergente ácida; EM: Energía metabolizable; NS: no significativo abc: literales que establecen diferencias entre las medias de los tratamientos.

Tabla 3. Producción y composición química de la leche de las dietas a base de P. purpureum CT-115 + concentrado comercial,marango fresco y ensilaje de marango

\begin{tabular}{|c|c|c|c|c|}
\hline & \multicolumn{4}{|c|}{ Tratamientos } \\
\hline Componenentes & $\begin{array}{l}\text { P. purpureum } \\
\text { CT-115 } \\
\text { concentrado } \\
\text { comercial }\end{array}$ & $\begin{array}{l}\text { Marango } \\
\text { fresco }\end{array}$ & $\begin{array}{c}\text { Ensilaje } \\
\text { de marango }\end{array}$ & $\begin{array}{c}\text { Error } \\
\text { estándar }\end{array}$ \\
\hline Leche (kg vaca día $\left.{ }^{-1}\right)$ & $13.92 \mathrm{a}$ & $13.65 \mathrm{a}$ & $12.65 \mathrm{~b}$ & 0.13 \\
\hline ECM (kg vaca día $\left.{ }^{-1}\right)$ & $13.07 \mathrm{a}$ & $12.90 \mathrm{a}$ & $11.90 \mathrm{~b}$ & 0.13 \\
\hline Grasa (g kg-1 de leche) & $34.88 \mathrm{a}$ & $35.33 \mathrm{a}$ & $35.05 \mathrm{a}$ & 0.17 \\
\hline Sólidos totales ( $\mathrm{g} \mathrm{kg}^{-1}$ leche) & $122.48 \mathrm{a}$ & $123.13 \mathrm{a}$ & $122.77 \mathrm{a}$ & 0.37 \\
\hline PB $\left(\mathrm{g} \mathrm{kg}^{-1}\right.$ de leche $)$ & $34.50 \mathrm{a}$ & $34.63 \mathrm{a}$ & $34.23 \mathrm{a}$ & 0.19 \\
\hline Caseína $\left(\mathrm{g} \mathrm{kg}^{-1}\right.$ de leche $)$ & $27.25 \mathrm{a}$ & $27.33 \mathrm{a}$ & $27.33 \mathrm{a}$ & 0.17 \\
\hline
\end{tabular}

ECM: Leche corregida por energía; PB: Proteína Bruta; NS: no significativo;

ab: literales que establecen diferencias entre las medias de los tratamientos.

Producción y composición de la leche.Los resultados obtenidos (tabla 3), muestran diferencias $(\mathrm{P}<0.01)$ para producción de leche $\left(\mathrm{kg}\right.$ vaca $\left.\mathrm{d}^{-1}\right)$ y leche corregida por energía ECM ( $\mathrm{kg}$ vaca $\left.\mathrm{d}^{-1}\right)$, sin embargo, esto no afectó la composición de la leche siendo similar entre los tratamientos.

El principal objetivo de este estudio fue observar si marango podía mantener el mismo nivel de producción que la dieta tradicional de $P$. purpureum y concentrado, permitiéndoles así a los productores obtener un alimento de alta calidad proteínica para sus vacas lecheras sin tener que comprar concentrados. Marango tiene un alto contenido de PB y en las dietas en que se diseñó cubrir loes requerimientos de energía de las vacas había un exceso de proteína, este exceso era altamente digestible como se observa en la tabla 2 , este exceso de proteína no condujo a un aumento de la producción de leche ya que el exceso de nitrógeno debe ser excretado en la orina a un costo energético para el animal. La alta digestibilidad de los nutrientes de marango, debe en teoría compensar ese costo extra de energía. De acuerdo con Oldham (1984), no se supone que vacas lactantes alimentadas con dietas que satisfacen los requerimientos de energía y proteína respondan con incrementos en producción de leche debido a incrementos en niveles de proteína bruta en la dieta.

Características organolépticas de leche y queso. Los resultados de la evaluación sensorial para características 
organolépticas de la leche (tabla 4), se observa diferencias significativas $(\mathrm{P}<0.001)$ para olor y sabor de la leche. Para queso (tabla 5) se indica igual tendencia. Los evaluadores encontraron que el queso elaborado a partir de ensilaje de Marango presentó la mayor aceptación para olor y sabor

Tabla 4. Características organolépticas de la leche analizada por jueces no entrenados

\begin{tabular}{|c|c|c|c|}
\hline \multicolumn{4}{|c|}{ Tratamientos } \\
\hline Características & $\begin{array}{l}\text { P. purpureum } \\
\text { CT-115+ } \\
\text { Concentrado } \\
\text { comercial }\end{array}$ & $\begin{array}{c}\text { Marango } \\
\text { fresco }\end{array}$ & $\begin{array}{c}\text { Ensilaje de } \\
\text { marango }\end{array}$ \\
\hline Color & $70 \mathrm{a}$ & $70 \mathrm{a}$ & $69 \mathrm{a}$ \\
\hline Olor & $90 \mathrm{a}$ & $57 \mathrm{~b}$ & 89 a \\
\hline Sabor & 89 a & $55 \mathrm{~b}$ & $91 \mathrm{a}$ \\
\hline Aspecto & $69 \mathrm{a}$ & $69 \mathrm{a}$ & $69 \mathrm{a}$ \\
\hline
\end{tabular}

$\mathrm{NS}=$ No Significativo; Escala numérica corresponde a la puntuación brindada por los jueces a cada uno de los tratamientos; ab: literales que establecen diferencias entre las medias de los tratamientos.

Tabla 5. Características organolépticas del queso analizado por jueces no entrenados

\begin{tabular}{|c|c|c|c|}
\hline \multicolumn{4}{|c|}{ Tratamientos } \\
\hline Características & $\begin{array}{l}\text { P. purpureum } \\
\text { CT-115+ } \\
\text { Concentrado } \\
\text { comercial }\end{array}$ & $\begin{array}{c}\text { Marango } \\
\text { fresco }\end{array}$ & $\begin{array}{c}\text { Ensilaje de } \\
\text { marango }\end{array}$ \\
\hline Color & $41 \mathrm{a}$ & $43 \mathrm{a}$ & $41 \mathrm{a}$ \\
\hline Olor & $45 \mathrm{a}$ & $29 \mathrm{~b}$ & $48 \mathrm{ab}$ \\
\hline Sabor & $39 \mathrm{~b}$ & $29 \mathrm{c}$ & $45 \mathrm{a}$ \\
\hline Textura & $40 \mathrm{a}$ & $40 \mathrm{a}$ & 39 a \\
\hline Apariencia & $40 \mathrm{a}$ & $41 \mathrm{a}$ & $40 \mathrm{a}$ \\
\hline
\end{tabular}

NS= No Significativo; Escala numérica corresponde a la puntuación brindada por los jueces a cada uno de los tratamientos; ab: literales que establecen diferencias entre las medias de los tratamientos.

Mucho se ha discutido sobre le efecto que tiene el marango sobre el sabor y olor de la leche. Agrodesierto (2010) recomienda suministrar follaje de marango al menos 3 horas antes del ordeño con el fin de evitar que la leche adquiera cualquier olor extraño, esta recomendación es, sin embargo, difícil de seguir en explotaciones con doble ordeño.

En este estudio se logra apreciar olor y sabor a hierba tanto en leche como en quesos elaborados a partir de vacas alimentadas con marango fresco como dieta única, esto puede ser explicado por los mecanismos de transmisión de olores a la leche reportados Dougherty et al., 1962 y Shipe et al., 1978 en los que afirman que la presencia de esteres en el alimento puede ser el responsable de olores atípicos en la leche. Bennet et al., (2003) reportó la presencia de concentraciones apreciables de 4-( $\alpha$-Lramnopiranosiloxi)- bencilglucosinolato y 3-cafeoilquinoico en las hojas de marango, estos esteres son los responsables del ya mencionado olor a hierba en leche. El ensilaje reduce hasta en un $90 \%$ las concentraciones de glucosinolatos (Nash 1985; Panciera et al., 2003) efecto que pudo notarse en los resultados presentados en las tablas 4 y 5 donde los jueces no reportaron diferencias significativas para leche $\mathrm{y}$ queso proveniente de tratamientos con dieta control o dieta a base de ensilaje de marango

Análisis financiero. El análisis financiero a través de los presupuestos parciales favorece al T2 (forrajero fresco de marango), ya que obtuvo una mayor utilidad, sin embargo, el efecto que tiene el uso de marango fresco en un $100 \%$ de la dieta sobre las características organolépticas de la leche, hace elegir al T3 (ensilaje de marango) como el más aceptable por no alterar dichas cualidades en la leche o el queso.

\section{CONCLUSIONES}

Los resultados obtenidos muestran claros indicativos de un buen aprovechamiento de los nutrientes del marango por parte de la vacas lecheras, lo que se expresa en producciones similares de leche y con características organolépticas aceptables, el claro ahorro con relación a la dieta convencional le brinda la importancia a este trabajo ya que permite presentarles a los productores una alternativa alimenticia de bajo costo y que se puede elaborar a partir de un recurso local que ellos tienen o pueden tener un su finca, además que permite promover la utilización racional de árboles y la siembra de especies forrajeras que mejoran el medio ambiente circundante.

Actualmente se impulsa a nivel nacional una estrategia para el fomento de la producción, consumo y comercialización del árbol de marango, como un insumo fundamental para contribuir agroecológicamente a la soberanía y seguridad alimentaria y nutricional a mediano plazo, en el marco de la crisis climatológica mundial. 


\section{REFERENCIAS BIBLIOGRÁFICAS}

Agrodesierto, 2010. Moringa, programas agroforestales. Revisado el 11 de octubre 2010 en http://www.agrodesiero.com/moringa.html AOAC. 1984. Official methods of analysis. Washington, DC, USA: Association of Official Analytical Chemists.

AOAC. 1990. Official Methods of Analysis. (12 th Ed.).Association of Official Analytical Chemists. Washington, D. C. 1018 p.

Bennet, R., Mellon, F., Foild, N., Pratt, J., Dupont, M., Perkins, L., Kroon, P. 2003. Profiling glucosinolates and phenolics in vegetative ans reproductive tissues of the multi-purpose tree Moringa oleifera. Journal of Agricultural and Food Chemistry, 51, 3456-3453

Dougherty, R., Shipe, W., Gudnason, V., Ledford, R., Peterson, R., Scarpellino, R. 1962. Physiological mechanisms involved in transmitting flavors and odors to milk. Journal of dairy science, 4, 472-476

FAO. 2010. Ganadería bovina en América Latina: Escenario 2008-2009 y tendencias del sector. Santiago de Chile.

Holmann, F., Rivas, L., Carulla, C., Rivera, B., Giraldo, L., Guzmán, S., Martínez, M., Medina, A., Farrow, A. 2003. Evolution of milk production systems in tropical Latin America and its interrelationship with the markets: an analysis of the Colombian case. Livestock Research for Rural Development 15.

INETER, 2006. Instituto Nicaragüense de estudios Territoriales. Estación Meteorológica del Aeropuerto Internacional Augusto Cesar Sandino, Managua.

Lindgren, E. 1979. Vallfodrets naringsvarde bestamt in vivo och med olika laboratoriemetoder. Report 45. Swedish University of Agricultural Sciences

Manh, L., Dung, N., Ngoi, T. 2005. Introduction and evaluation of Moringa oleifera for biomass production and as feed for goats in the Mekong Delta. Livestock Research for Rural Development 17.

McDonald, P., Edwards, R., Greenhalgh, J. 1988. Animal Nutrition, fourth ed. Longman group, United Kingdom

Mendieta-Araica, B., Sporndly, E., Reyes-Sánchez, N., Norell, L., Sporndly, R. 2009. Silage quality when Moringa oleifera is ensiled in mixtures with Elephant grass, sugar cane and molasses. Grass and Forage Science. 64. 364-373.

Nash, J. 1985. Crop conservation and storage in cool, temperate climates (Pergamon Press, Oxford)

NRC. 1988. Nutrients requirements of dairy cattle. Sixth revised edition. National Academy of Science. Washington, USA

Oldham, J. 1984. Protein-Energy interrelationships in dairy cows. Journal of Dairy Science, 67, 1090-1114.

Panceira, M., Kunkle, W., Frnsen, S. 2003. Minor silages crops, in: Buxton, D. Silage science and technology. American Society of Agronomy. Madison

Pastor, L., Mellado, B., Ramírez, A.., Dolores R.., 2008. Evaluación sensorial de queso de leche de cabra tipo Bour sin sabor natural y ceniza (Sensory evaluation of goat milk cheese type boursin natural and ash flavor). http://www.Revista Electrónica de Veterinaria.

Patterson, H., Lucas, H. 1962. Change-over designs. North Carolina Agr. Exp. Sta. Tech. Bull. 147 p.

Pérez, J. 1993. Pautas básicas para el análisis financiero de proyecto agropecuario en inversión para pequeñas empresas rurales. Manual de capacitación para técnicos de campo. IICA, San José, Costa Rica. 292 p

Reyes-Sanchez, N., Sporndly, E., Ledin, I. 2006. Effect of feeding different levels of foliage of Moringa oleifera to creole dairy cows on intake, digestibility, milk production and composition. Livestock Science, 1001, 24-31

SAS. 2004. SAS/STAT User's Guide Version 9.1. Cary, NC, USA: SAS Institute Inc.

Shipe, W., Váhasete, E., Deane, D., Dunkley, W., Hammond, E., Harper, W. 1978. Off flavor of milk: nomenclature, standards and bibliography. Journal of Dairy Science. 61, 855-869.

Steinfeld, H., Gerber, P., Wassenaar, T., Castel, V., Rosales, M., de Haan, C. 2006. Livestock`s long shadow: environmental issues ans options, (FAO, Rome)

14. Undersander, D., Mertens, D.R. y Thiex, N. 1993. Recommends forage analyses procedure. National Forage Testing Association, USA.139 p. (en línea), Consultado el 28 de octubre 2009.Disponible en URL: http://www.foragetesting.org/files/LaboratoryProcedures. pdf.

SAS. 2004. SAS / STAT User's Guide Version 9.1. Cary, NC, USA: SAS Institute Inc.

Undersander, D., Mertens, D.R. y Thiex, N. 1993. Recommends forage analyses procedure. National Forage Testing Association, USA.139 p. (enlínea), Consultado el 28/10/09.Disponible en URL: http://www.foragetesting.org/files/LaboratoryProcedures.pdf.

Van Soest, P., Robertson J., Lewis B. 1991.Methods for dietary fibre, neutral-detergent fibre and non-starch polysaccharides in relation to animal nutrition. Journal of Dairy Science, 4, 3583-3597. 